\title{
Expérience carcérale et procès social: $U n$ roman français de Frédéric Beigbeder ou le discours auto(socio)biographique d'un marginal cocaïnomane
}

\section{Jail experience and social trial: Frédéric Beigbeder's $U n$ roman français or the auto(socio)biographical discourse of a marginal cocaine addict}

\author{
Armel Jovensel NGAMALEU' ${ }^{1}$ (1)
}

${ }^{1}$ PhD student, University of Douala, French and Francophone Studies, Douala, Cameroun

ORCID: A.J.N. 0000-0002-5448-6689

Corresponding author: Armel Jovensel NGAMALEU, Université de Douala, Français et Études Francophones, Douala, Cameroun E-mail: jovenselngamaleu@gmail.com

Submitted: 02.02.2020

Accepted: 06.03.2020

Citation: Ngamaleu, A. J. (2020) Expérience carcérale et procès social : Un roman français de Frédéric Beigbeder ou le discours auto(socio)biographique d'un marginal cocaïnomane. Litera, 30(1), 177-197.

https://doi.org/10.26650/LITERA2020-0007

\section{RÉSUMÉ}

Bon nombre d'écrivains ont vécu l'expérience carcérale pour des motifs différents. Nous pouvons citer, notamment en France, François Villon, Maquis de Sade, Guillaume Apollinaire, Paul Verlaine, Jean Genet et Frédéric Beigbeder. Ce dernier est une personnalité médiatique dans son pays, précisément depuis la publication, en 2000, de son roman 99 Francs devenu un best-seller. Cependant, dans la nuit du 28 janvier 2008, Beigbeder a été pris en flagrant délit de consommation de cocaïne sur le capot d'une Bentley, en pleine rue, lors d'une soirée festive, avec un groupe d'amis artistes. Alors, il sera arrêté par la police et encellulé avec un ami, l'écrivain français Simon Liberati. Les autres noceurs échapperont aux policiers. C'est pendant les trois jours de sa détention que Beigbeder sera confronté au travail de la mémoire, à travers les souvenirs de son passé, notamment de son enfance, pour reconstruire son identité et essayer de défendre sa posture d'écrivain marginal (Meizoz, 2011). Prix Renaudot 2009, Un roman français est un récit autobiographique dans lequel l'auteur a enfin pris la résolution de décadenasser sa personnalité atypique pour mieux justifier sa vision du monde tout en pointant un doigt accusateur sur sa société. Car, à son avis, il n'est que le fruit de l'arbre qu'est la France. Son histoire individuelle/personnelle est ainsi mise en relation avec I'histoire sociale ou les réalités sociopolitiques en France. C'est d'ailleurs ce qui peut justifier le choix du titre métaphorique et thématique de son œuvre autobiographique.

Mots-clés: Discours auto(socio)biographique, expérience carcérale, procès social, cocaïne, libert

\section{ABSTRACT}

Many writers have gone through the experience of a jail sentence for various reasons. Among others, mention could particularly be made of François Villon, Maquis de Sade, Guillaume Apollinaire, Paul Verlaine, Jean Genet and Frederic Beigbeder, all from France. The latter is a highly publicized personality in his country, 
especially since the publication of his novel 99 Francs became a best-seller in 2000. However, on the night of January 28, 2008 , Beigbeder was caught in the act of consuming cocaine on the hood of a Bentley in the street on a festive night, with a group of artist friends. So he was arrested by the police and jailed with a friend, the French writer Simon Liberati. The other revellers escaped the police. It was during the three days of his detention that Beigbeder was confronted with the work of memory, through the memories of his past, especially his childhood, to rebuild his identity and try to defend his posture as a marginal writer (Meizoz, 2011). The winner of the Prix Renaudot 2009, Un roman français is an autobiographical narration in which the author finally decided to explore his atypical personality to better justify his vision of the world while pointing an accusing finger at his country. This is because, in his opinion, it is only the fruit of the tree that is France. His individual/ personal history is thus related to social history or socio-political realities in France. This is what can justify the choice of the metaphorical and thematic title of his autobiographical novel.

Keywords: Auto(socio)biographical discourse, jail experience, social trial, cocaine, freedom

\section{EXTENDED ABSTRACT}

Many writers have experienced a period of time in jail for different reasons. Among others mention could particularly be made of François Villon, Maquis de Sade, Guillaume Apollinaire, Paul Verlaine, Jean Genet and Frederic Beigbeder, all from France. The latter is a highly publicized personality in his country, especially since the publication of his novel 99 Francs became a best-seller in 2000. However, on the night of January 28, 2008 , Beigbeder was caught in the act of consuming cocaine on the hood of a Bentley in the street on a festive night, with a group of artist friends. So he was arrested by the police and jailed with a friend, the French writer Simon Liberati. The other revellers escaped the police. It was during the three days of his detention that Beigbeder was confronted with the work of memory, through the memories of his past, especially his childhood to rebuild his identity and try to defend his posture as a marginal writer (Meizoz, 2011). The winner of the Renaudot prize in 2009, Un roman français, is an autobiographical narration in which the author finally decided to explore his atypical personality to better justify his vision of the world while pointing an accusing finger at his country. This is because, in his opinion, it is only the fruit of the tree that is France. His individual/personal history is thus related to social history or socio-political realities in France. Therefore, this article questions the nature and the contours of the relationships that the narrator-hero of Un roman français maintains with his society, France. In other words, we are trying to understand and explain or justify the logics underlying these dialectical relationships. The matrix and illustrative element of the antagonism between the novelist Beigbeder and his native country is his arrest and his imprisonment. Indeed, the case of Frédéric Beigbeder illustrates the fact that the individual, out of pure selfishness and pride inherent in human nature, tends to make private choices and behave in a transgressive manner, to the detriment of society. Life in society is governed 
by laws, rules and the moral and ethical principles that guide and condition human actions. Consequently, each individual is bound by the conventions and values that govern their social universe. However, in Un roman français there is a break, almost a radical divorce between the microcosm that is the individual subject and the macrocosm that is the social subject. Thus, the narrator-hero-author Frédéric Beigbeder proceeds, in his autobiographical project, to a staging of himself under the prism of the social realities with which he is confronted. It turns out that Beigbeder is a libertarian, individualistic and hedonistic individual. He starts from his painful prison experience to denounce the conditions of detention in France, while expressing his disdain for his native country in a resolutely pamphleteer tone. So, it goes without saying that the novelist is completely out of phase with ultra-capitalist, paternalistic and moralistic France, which is properly pathological and therefore agonizing, even alienating. It is important to note that France is targeted by the author from its metaphorical title: Un roman français. Beigbeder, speaking of his individual self in close relation to the social self, manages to accuse his native France which is, in his opinion, a liberticidal society because it stifles or limits the development of the individual trapped in an alleged "freedom". According to the writer, his country is not a F/freedom land. Upon reading this title, Frédéric Beigbeder's life would be the (more or less broken) mirror of French society. The writer sees himself as a pure and hard product of his society: he correlates his life, his personal history with the collective experience. In other words, Beigbeder's autobiographical project problematizes the individual through society. In this sense, his initiative is part of the regime of satirical autosociobiographism. 


\section{Introduction}

La société est le cadre de vie où l'individu est appelé naturellement à évoluer. L'homme, on le sait, est un être né pour vivre en société en tant que maillon de la chaîne sociale. Cependant, le rapport individu-société est loin d'être un long fleuve tranquille. De fait, la vie en société est régie par les lois, les règles ainsi que les principes moraux et éthiques qui guident et conditionnent les actions humaines. Par conséquent, chaque individu est tenu par les conventions et les valeurs qui régissent son univers social. Alors, si la relation entre l'individu et la société est une alliance naturelle (Aristote, 1874) ou contractualiste (Hobbes, 2002 ; Rousseau, 2012), elle demeure, néanmoins, problématique. Car les désirs et les passions varient d'un individu à l'autre. Ainsi, la société, en réalité, constitue un théâtre d'expressions, d'actions et d'aspirations diverses et antagonistes. C'est ce qui crée les tensions qui, en conséquence, alimentent la dynamique sociale. Dès lors, en dépit ou à cause des conventions et contraintes sociales, I'homme entretient avec la société un rapport dialectique, ambivalent ou mitigé. L'œuvre autobiographique de Frédéric Beigbeder illustre à bien d'égards cet état de chose. D'ailleurs, le contexte d'écriture de cette autobiographie est lié à une violation par l'auteur cocaïnomane de la loi en matière de consommation de drogue illicite en France. À cet effet, cette réflexion envisage d'interroger la nature et les contours des rapports que le narrateur-héros d'Un roman français entretient avec sa société, la France. Autrement dit, nous voulons comprendre et expliquer ou justifier les logiques qui sous-tendent ces rapports dialectiques. L'élément matriciel et illustratif de l'antagonisme entre le romancier Beigbeder et son pays natal est son arrestation et son incarcération. N'étant pas outillé pour écrire dans sa cellule ${ }^{1}$, Un roman français sera donc écrit aux lendemains de cette incarcération et publié en 2009 aux Éditions Grasset \& Fasquelle. Dès lors, en quoi Un roman français met-il en évidence l'expression de l'expérience carcérale et le procès social de Frédéric Beigbeder ? Nous nous proposons de répondre à cette question en montrant d'abord la manifestation ou la célébration de la mondanité chez Frédéric Beigbeder. Ensuite, nous analyserons comment du fait de son illusion hédoniste

1 Le romancier revient sur cette situation lors d'une interview accordée à Jérôme Colin (2010, p. 7) : « Ben, lorsque vous êtes dans une cellule, il y a la claustrophobie qui donne envie de s'évader. Donc, c'est le moment où je me suis dit - tiens, c'est bizarre, je ne me souviens de rien, je ne me souviens pas de mon enfance, à force de l'occulter, je l'ai effacée. Et donc, j'ai commencé à penser que c'était peut-être un sujet de livre. Au lieu de raconter tout le temps des histoires de mec bourré de fric, qui drague des mannequins dans des boîtes de nuit, je vais raconter l'histoire d'un petit garçon, avec son grand-père, sur une plage dans le Sud-Ouest de la France, qui saigne du nez parce que ses parents divorcent. Un truc finalement moins spectaculaire, peut-être plus chiant, mais plus intime, plus vrai et plus sincère. Et j'étais persuadé que je ne le faisais que pour moi et que ça n'intéresserait personne. » 
Beigbeder viole le « contrat social » (Rousseau, 2012) et sera puni à cet effet par la règlementation en vigueur. Enfin de compte, nous nous intéresserons aux enjeux du procès social fait par le romancier, suite à sa désillusion révoltante.

\section{La célébration des milieux mondains : réjouissance et délire}

La mondanité est en toile de fond de l'autobiographie de Frédéric Beigbeder qui est un prototype de l' « homo festivus ». D'après Lakis Proguidis (2002, p. 65), nous vivons dans « l'ère festive ", car "l'on s'adonne aux parties de plaisir et aux fêtes nocturnes ». «L'idée est que nos sociétés ont massivement opté pour la « Glucocratie »(du grec glucos, sucré), pour le kitsch, pour l'embellissement par le camouflage » (id.). L'individu se pervertit dans la quête du plaisir immédiat ; bref, du sucré, de l'excitant, du jouissif. Autant souligner que « [l'homo festivus] ne jouit pas; il est l'incarnation de la jouissance. II ne fête pas ; il est l'hypostase de Dionysos » (ibid., p. 47). De fait, « [...] I'homme contemporain [a] besoin d'être toujours ailleurs, d'être transporté et enveloppé dans une ambiance syncopée, comme s'il avait besoin d'une déréalisation stimulante, euphorique et enivrante du monde » (Lipovetsky, 1983, p. 33).

Le narrateur-auteur dans Un roman français a un rapport heureux avec certains espaces qui sont sources d'euphorie pour lui. Frédéric Beigbeder est, d'emblée, un noceur noctambule. Pour cette raison, il affectionne des lieux de festivités nocturnes. C'est un oiseau de nuit. II se livre donc avec ses compagnons noceurs aux plaisirs des festivités, en consommant de l'alcool, de la cigarette et de la cocaïne. C'est en des termes teintés d'orgueil et de snobisme que l'auteur relate, de manière émerveillée, leur soirée de noceurs:

Je fredonnais Where is my mind des Pixies sur mon scooter. J'étais déguisé en lycéen, chaussé de boots camarguaises en daim, cheveux mi-longs en bataille, cachant mon âge dans ma barbe et mon imperméable noir. Je pratique ce genre de dérive nocturne depuis plus de vingt ans, c'est mon sport favori, celui des vieux qui refusent de vieillir. Pas facile d'être un enfant prisonnier dans un corps d'adulte amnésique. (Beigbeder, 2009, p. 15)

Le passage laisse percevoir manifestement tout le plaisir ressenti par le narrateur dans cette atmosphère chaleureuse, détendue, sympathique et festive. «La soirée avait bien commencé ", s'en souvient-il. À cet effet, i l énumère les actions au menu ce soir-là : « dîner arrosé de grands crus », « tournée habituelle de bars tamisés » et «consommation 
de shots multicolores ». La soirée festive entre amis connote l'aisance, le snobisme et la désinvolture. Le narrateur-noceur décrit cette soirée avec une coloration poétique, s'inspirant de la poésie rimbaldienne. De plus, il attise sa joie en fredonnant la chanson «Where is my mind » des Pixies, assis sur son véhicule motorisé. Le titre de cette chanson en dit long sur le désir de l'évasion du personnage, par une noyade ou une fonte de sa mémoire dans la musique, l'alcool et sans doute la drogue. II perd donc le sens de toute responsabilité et de la maturité pour se déguiser en un jeune inconséquent. Il se prend pour un fêtard juvénile en se livrant à sa « dérive nocturne ", à son vieux « sport favori », commencé depuis son adolescence et qui est devenu(e) sa parfaite passion. II se plaît à jouer à la folie juvénile comme s'il avait perdu la raison. Mais, c'est simple : il est en quête de plaisir éphémère. Frédéric Beigbeder est, en réalité, un être d'un tempérament jouisseur. Il s'y est habitué depuis sa jeunesse. « Je pratique ce genre de dérive nocturne depuis plus de vingt ans [...] », reconnaît-il, vaniteusement. Or I'habitude a la peau dure. Cette frasque est la sienne depuis sa vie de jeunesse à New-York, ville qui symbolise la liberté pour le héros.

De plus, l'avenue Marceau, cette avenue célèbre à Paris, constitue le théâtre de la réjouissance vicieuse des noctambules fêtards. Le groupe des vieux délinquants qui refusent de vieillir, dans lequel se trouve Beigbeder, prend du plaisir en transgressant la loi pour se livrer dans cette rue à son vice. Le narrateur avoue notamment : « Depuis un mois, une nouvelle loi républicaine interdisait de fumer à l'intérieur des discothèques, un attroupement s'était formé sur le trottoir de l'avenue Marceau » (Beigbeder, 2009, p. 15). Nous constatons qu'à défaut de se regrouper dans des discothèques, Frédéric Beigbeder et ses acolytes s'attroupent dans la rue pour se donner (librement ?) à leur vice. Le trottoir devient le théâtre de leurs manifestations nocturnes et cyniques. L'avenue Marceau symbolise l'expression de leur désinvolture, de leur insouciance ou immaturité. Elle constitue un lieu euphorique pour la bande de noceurs. Ce dernier décrit ses actions lors de cette soirée festive, en compagnie de sa " génération insomniaque », son groupe de fêtards :

J'étais un non-fumeur solidaire des jolies filles sur escarpins vernis qui se penchaient vers les briquets tendus. [...] Je tenais un verre dans une main, de l'autre je m'accrochais à des épaules fraternelles. Je baisais la main d'une serveuse en attente d'un rôle dans un long métrage, tirais les cheveux d'un rédacteur en chef de magazine dénué de lecteurs. (Beigbeder, 2009, p. 15) 
Le groupe de noceurs noctambules parvient enfin à l'apothéose de ses réjouissances par la consommation de la cocaïne. En effet, ce sont des alcooliques et cocaïnomanes qui s'adonnent à leur plaisir, devenu une véritable passion pour eux, dans la rue, en toute liberté. La rue, notamment l'avenue Marceau, représente un refuge pour ces jouisseurs qui se plaisent dans la transgression de la loi. Nous comprenons que la nuit est un moment propice pour ces jouisseurs de pratiquer leurs vices librement. La rue et autres lieux de débauche constituent le théâtre de leur euphorie. L'intervention de la police est un réel cauchemar pour ces délinquants. Elle vient crisper l'ambiance euphorique qui régnait jusque-là entre ces amis cocaïnomanes. Les policiers viennent troubler leur fête et parviennent à mettre la main sur Frédéric Beigbeder et son ami le Poète. C'est la descente aux enfers des geôles.

\section{La violation de la loi ou la confusion entre liberté littéraire et liberté civile : la descente aux enfers des geôles}

La gestion ou l'appréhension de la liberté chez un individu peut être fonction de la profession exercée. La relation profession-liberté mérite de ce fait d'être analysée dans l'autobiographie de Frédéric Beigbeder qui est un écrivain attitré, consacré et célèbre. Pour John Stuart Mill, dans son ouvrage De la liberté, le concept de liberté prend en compte : « [ ] les rapports de la société et de l'individu dans tout ce qui est contrainte ou contrôle, que les moyens utilisés soient la force physique par le biais de sanctions pénales ou la contrainte morale exercée par l'opinion publique » (Mill, 1990, p. 11). Il s'ensuit que l'individu fait partie de la chaîne sociale et, qu'il n'est pas appelé à ramer à contre-courant, peu importe son statut socioprofessionnel. De fait, il doit agir suivant certains canons sociaux ou moraux. Dans le cas contraire, il met sa liberté civile en péril. Car, il existe des « sanctions pénales » et «la contrainte morale exercée par l'opinion publique ». C'est dire que l'individu est un microcosme qui évolue dans le macrocosme social ; il doit par conséquent se conformer pour ne pas transgresser les règles et conventions régissant le jeu social. C'est d'ailleurs pourquoi Jean-Jacques Rousseau (2012, p. 76), théoricien contractualiste, écrit : « L'obéissance à la loi qu'on s'est prescrite est liberté ».

Toutefois, I'homme est un animal naturellement égoïste. II préserve ses intérêts ou tout ce qui est susceptible de lui faire plaisir au détriment des autres et donc de la société. Certes, un citoyen n'est point censé ignorer les lois de sa société ; mais, il est animé par une volonté transgressive. Il veut toujours passer outre les normes, franchir les limites 
ou contraintes afin de mieux s'exprimer, notamment sous le prisme de sa profession. C'est dans cette perspective que s'inscrit le cas Beigbeder, au regard de la perception et la gestion de sa liberté. Son sens de la liberté est en rapport étroit avec son statut d'écrivain « people ${ }^{2}$, pour reprendre son terme qualificatif. En d'autres mots, il s'avère que le narrateur « autodiégétique » (Genette, 1972) est un écrivain qui exprime sa liberté civile en fonction de sa liberté de création. En effet, Frédéric Beigbeder est une personnalité mondaine et libertine. Il se livre à un jeu de mondanité avec ses amis et collègues artistes. Alors, ils se donnent le luxe de passer outre la loi, ostensiblement. Ils ont été pris en flagrant délit de consommation de stupéfiant sur le capot de leur voiture, donc sur la voie publique. C'est, bien entendu, leur arrestation par la Police française et leur garde à vue, qui réveillera en eux la nature même de la liberté. C'est-à-dire une réalité qui a des facteurs limitants. Ainsi, Beigbeder pense que, puisque son ami (le Poète) ${ }^{3}$ et lui sont des écrivains (célèbres) en France, ils devraient se permettre de faire ce qu'il veut. Leur titre d'« écrivain » est une source d'illusion pour lui précisément. Nous pouvons écouter leur désaccord face à l'Ordre public dans le passage fort significatif suivant :

Le Policier : - Mais qu'est-ce qui vous prend de faire ça sur une voiture? Le Poète : - La vie est un CAUCHEMAR !

Moi : - Je descends d'un homme crucifié sur des barbelés de Champagne ! [...]

Un autre Policier : - Vous êtes dingues de faire ça sur la voie publique, planquez-vous aux chiottes comme tout le monde ! C'est de la provocation, là !

Moi (en essuyant la poudre sur le capot de la voiture avec mon écharpe) :- Nous ne sommes pas tout le monde, mon commandant. Nous sommes des ZÉCRIVVAINS. OKAY?

[...]

Le Poète (avec force mouvements de tête supposés indiquer la dignité humaine et l'orgueil de l'artiste incompris) : — La liberté est impossssible... . [sic]

[...]

Le Chef : - Oh lala, ça sent la garde à vue ! Allez zou, coffrez-moi tout ça !

Moi :- Mais... mon frère à la Légion d'honneur ! (Beigbeder, 2009, pp. 21-22)

\footnotetext{
C'est-à-dire très connu, très médiatisé.

3 Il est désigné comme tel dans le roman.
} 
Face aux policiers, le Poète se montre « fayot et crâneur à la fois », reconnaît Beigbeder. II clame ses origines nobles. II s'aveugle éperdument face au délit commis et met en relief son statut d'écrivain. « Nous ne sommes pas tout le monde, mon commandant. Nous sommes des ZÉCRIVVAINS. OKAY ? ", souligne-t-il avec fierté4. Ils se passent, son collègue le Poète et lui, pour des artistes « incompris ». Ils sont en déphasage avec la réalité. Ils vivent dans l'illusion que leur confère le titre $d^{\prime}$ " écrivain ». Le Poète et Beigbeder dans cette scène sont l'illustration tangible de l'artiste incompris par leurs semblables. La didascalie liée à la réplique du poète, précise d'ailleurs cette posture qui particularise la figure de l'artiste dans la cité. On est en droit de penser au célèbre poème, "L'Albatros », de Charles Baudelaire (2001) dans Les Fleurs du Mal. L'artiste semble décidément être un homme des nuées ; un homme qui sort de l'ordinaire ou qui ne s'accommode pas facilement des conventions sociales. Les artistes, notamment les écrivains, s'avèrent être, pour la plupart, des anticonformistes en ce sens qu'ils brillent parfois par leur marginalité. Dans cette perspective, l'artiste est perçu comme un réfractaire. Jean Cocteau, poète français, estimait que le poète, et par extension l'artiste, est celui-là qui « met les pieds dans le plat ». En d'autres termes, l'artiste ne se conforme pas toujours aux normes en vigueur non seulement sur le plan artistique, mais aussi sur le plan social. Néanmoins, force est de constater que l'artiste demeure impuissant face aux contraintes ou au déterminisme de la société. Tel est le cas pour le duo Beigbeder et le Poète.

Frédéric Beigbeder semble se prendre pour la plaque tournante ou le nombril du monde. Son nombrilisme et sa mégalomanie trahissent cette volonté de sa part à être traité différemment, à être considéré comme un citoyen à part. Mais il oublie que la loi est impersonnelle. Il ne saurait donc être au-dessus de la loi. La formule latine le prouve bien : dura lex, set lex, « la loi est dure, mais c'est la loi ». Elle s'abat sur le coupable et s'applique conformément et objectivement aux textes en vigueur, sans particularisme. Or, Beigbeder est un auteur nombriliste qui se voit tel un citoyen particulier, spécial ; en fait, il est fier d'être un écrivain célèbre.

Toutefois, il va vivre une désillusion singulière et douloureuse dans l'univers carcéral. D’ailleurs, le titre de son premier chapitre en dit long à ce propos : « Les ailes coupées ». C'est une métaphore animalière qui traduit la déchéance de Frédéric Beigbeder. L'écrivain

4 Le caractère typographique témoigne, à l'écrit, de l'intention de mise en relief du titre ou du statut social de ces intellectuels marginaux. À l'oral, puisqu'il s'agit bien d'un dialogue, on peut s'imaginer l'intonation du locuteur coupable et provocateur Beigbeder. 
« people » se compare à un simple oiseau qui n'a plus ses ailes pour fendre l'air à sa guise, errer imperturbablement. Beigbeder était jusqu'à cette arrestation et incarcération un véritable oiseau de nuit, un « King of the night », pour reprendre son expression, qui se livrait à tous les excès nocturnes (alcool, drogue, sexe, etc.). Il est en effet quitté de héros à zéro, de personnalité à simple individu (pas différent des autres détenus), bref du paradis à l'enfer. Cette descente aux enfers des geôles est d'autant plus manifeste dans ce passage de l'incipit :

Le 28 janvier 2008, au commissariat du VIlle arrondissement de Paris, des fonctionnaires en uniforme bleu, revolver et matraque à la ceinture, me déshabillaient entièrement pour me fouiller, confisquaient mon téléphone, ma montre, ma carte de crédit, mon argent, mes clés, mon passeport, mon permis de conduire, ma ceinture et mon écharpe, prélevaient ma salive et mes empreintes digitales, me soulevaient les couilles pour voir si je cachais quelque chose dans mon trou du cul, me photographiaient de face, de profil, de trois quarts, tenant entre les mains un carton anthropométrique, avant de me reconduire dans une cage de deux mètres carrés aux murs couverts de graffitis, de sang séché et de morve. (Beigbeder, 2009, p. 10)

Dans sa cellule, il se surprend de l'atmosphère qui y règne. Pourtant, le climat bruyant, invivable du milieu carcéral qu'il déplore n'est pas pour autant un phénomène étrange. Il écrit : «Dans la cellule voisine, un pickpocket tapait du poing sur la vitre sans conviction, mais avec suffisamment de régularité pour interdire tout sommeil aux autres détenus. S'endormir eût été de toute façon utopique [ ] » (Beigbeder, 2009, p. 10). Il a été en infraction, il a été arrêté et détenu ; il devrait subir, vivre sa peine en homme mature et responsable afin d'assumer ses actes. Mais, hélas, ce n'est pas le cas chez lui ; il revendique une reconnaissance de sa part, en vain. Le narrateur est incarcéré avec son collègue et ami le Poète. C'est un autre désinvolte ; un jouisseur au même titre que Beigbeder, son compagnon dans la débauche. II exprime son soulagement lorsque l'occasion de leur rencontre se présente. II s'exclame comme un enfant à la vue de ce dernier :

Bonne nouvelle : je retrouve le Poète ! II a enfin dessoûlé. Il a une haleine de vodka vieille d'une nuit sans brossage de dents, odeur que nous qualifierons de : « vodkaïnée ». Il ne se souvient de rien, ni de l'arrestation, ni de notre fuite piteuse, ni de la nuit de cauchemar enfermé sous la terre. 
II me raconte que la police a perquisitionné dans son appartement avec des chiens junkies. Ils n'ont rien trouvé mais les pauvres animaux en manque reniflaient la table à l'endroit où il saupoudre habituellement du produit ! (Beigbeder, 2009, p. 81)

Le Poète est son miroir. II reflète leur désinvolture, car tous deux sont des écrivains jouisseurs. Leur bêtise est partagée, de même que leur irresponsabilité. Ce sont des consommateurs de stupéfiants avérés. La fouille de la maison du Poète montre que ce dernier en consomme non seulement dans la rue, mais aussi en privée en toute quiétude. Les souris de son appartement en ont, elles-aussi, pris goût. II détient trois grammes de stupéfiant sur lui au moment de l'arrestation; ce qui rend sa situation plus précaire. Mais, comme le dit le narrateur, « le pessimisme lui sert d'armure ». En d'autres mots, il ne redoute plus le mal ; le risque et le pire font partie de son être, de sa personnalité. Mieux, nous avons affaire à un psychopathe. Beigbeder n'est pas épargné ; il est, lui aussi, un malade ; tous deux souffrent de la quête des plaisirs incontrôlés, quitte à transgresser la loi. C'est pourquoi, ils cèdent aux caprices du corps dans l'optique de satisfaire leur penchant libertin. Ils font fi des lois en vigueur :

Il y avait [...] un acteur de cinéma d'auteur, quelques chômeuses, des videurs noirs et blancs, un chanteur démodé et un écrivain dont j'avais publié le premier roman. Quand ce dernier a sorti un sachet blanc pour verser de la poudre sur le capot d'une Chrysler noire qui scintillait dans la contre-allée, personne n'a protesté. (Beigbeder, 2009, p. 15)

Nous remarquons que l'esprit libertin et cynique est une commodité dans le milieu des artistes. Car les personnes évoquées par le narrateur, ayant pris part à la scène coupable, sont des artistes (acteur de cinéma, chanteur et écrivain). Ils s'engagent ce soir-là, d'après le récit du narrateur, à violer la loi selon leur gré. Le constat est amer : des intellectuels, voir des modèles deviennent des anti-modèles, des rebelles ou des délinquants. Ils outrepassent la norme afin de se faire plaisir et d'assouvir leurs désirs de toxicomanes. On note ainsi, un goût et un plaisir à la transgression. Transgresser devient un acte de liberté et de bravoure ou d'héroïsme ; une manière de s'exprimer et donc d'exister. Dans ce sillage, vivre ne se résume plus à la contemplation et au respect des normes et conventions sociales en vigueur; mais l'acte de vivre sous-tend la capacité à braver les obstacles qui compromettent l'épanouissement, la jouissance. Ainsi, peut-on dire qu'il existe un plaisir dans la transgression. Et, la fierté de transgresser importe plus que la conscience d'une peine ultérieure. Le principe cardinal semble 
donc être de jouir d'abord, quitte à souffrir après. C'est la règle du jeu des jouisseurs. Frédéric Beigbeder avoue : "Braver la loi nous amusait ; nous vivions des temps de Prohibition, il était l'heure de désobéir comme Baudelaire et Théophile Gautier, Ellis et Mc Inerney, ou Blondin que Nimier venait délivrer du commissariat déguisé en chauffeur de maître » (Beigbeder, 2009, p. 15).

De ce fait, il s'avère que la gestion de la liberté chez Beigbeder, tout comme chez ses amis artistes, est influencée par sa profession. Il a le désir, voire la passion manifeste d'une jouissance exacerbée de la liberté. Il a tendance à confondre liberté et libertinage car il semble ne plus distinguer la fiction, lieu de l'expression libre des phantasmes vicieux ou cyniques, et la société réelle, lieu des normes et conventions inaliénables. Nous remarquons avec force que chez Frédéric Beigbeder, la liberté civile, dont doit, en principe, jouir chaque citoyen normal est distendue, voire dénaturée à cause de sa posture d'écrivain. Car ce dernier semble confondre l'univers fictionnel régi par la liberté de création littéraire (pratiquement illimitée) et l'univers réel ou social régi par la liberté civile (toujours limitée). Beigbeder est donc une personne mégalomaniaque, hédoniste et irresponsable. C'est certes un écrivain-bourgeois ${ }^{5}$ attitré, consacré et célèbre ; mais, il est un esprit rebelle et marginal qui déploie son nombrilisme et son cynisme, avec un orgueil flagrant et provocant. L'expérience carcérale va lui permettre de se désillusionner amèrement. Car, il sera confronté à une réelle dysphorie troublante et humiliante dans l'univers pénitentiaire. Cette douloureuse expérience carcérale va traumatiser et révolter Beigbeder. Il s'engage subséquemment par le biais de l'écriture à faire un vitriol de sa France natale.

\section{Du procès de la société française}

La détention de Beigbeder l'a autant humilié qu'indigné. En réaction, contre cet affront, il s'engage à faire une diatribe de sa société. En effet, Beigbeder dénonce le caractère contraignant, liberticide et donc suicidaire de la société française. Son divorce avec sa société est prononcé en ces termes : «[...] j'expose mon désaccord avec une société qui prétend protéger les gens contre eux-mêmes, les empêcher de s'abîmer,

5 Le philosophe français Philippe Muray privilégie le terme familier « bobo » pour désigner le bourgeois qui, en réalité, mène une vie dissolue/vicieuse et vaniteuse. Selon Muray, «le bobo ne vit que tourné autour de son propre plaisir, de son accession à une forme de supériorité intellectuelle, sociale et culturelle à défaut d'être financière. Il est une sorte d'enfant-roi autour duquel tout tourne » (Fabrice Luchini, 2009, p. 97). Force est de constater que Frédéric Beigbeder, notre cas d'étude, illustre à suffire ces propos ; puisqu'il constitue le parangon du «bobo », en tant que, de surcroit, un être festif (« homo festivus ») mégalo-snob et narcissique. 
comme si l'être humain pouvait vivre autrement qu'en collectionnant des vices agréables et des addictions toxiques » (Beigbeder, 2009, p. 43). II va sans dire que Beigbeder se veut un libertin/libertaire ou un anarchiste. Dans la mesure où il est contre le système normatif social. II conçoit que l'individu doit être responsable de ses propres choix et les assumer personnellement sans l'intervention de la loi, sans l'oppression ou la contradiction d'une quelconque autorité. Dans la préface de l'ouvrage Drogues: pourquoi la légalisation est inévitable de Michel Henry, Beigbeder, le préfacier, écrit :

Le pire dans ce gâchis monumental, c'est l'évolution des démocraties vers un paternalisme étatique attentatoire aux libertés. On l'a vu à propos de la cigarette : nous sommes entrés dans une dictature de la santé. Le principe de précaution est devenu un principe de protection. Je ne demande pas à l'État de me protéger : mon pays n'est pas un préservatif géant ! [ ] la société du "Care » est une absurdité fasciste. L'interdiction des drogues est une erreur politique, économique, sociale et humaine aux dégâts considérables. Elle place la police dans des situations intenables : obligée de contrôler tout citoyen qui s'amuse, de le menotter, de l'enfermer au lieu de courir après les violeurs et les assassins. [ ] Quand on commence à arrêter les citoyens pour leur bien, où est la limite ? Les camps de rééducation voulaient le bien du peuple cambodgien, les hôpitaux psychiatriques soviétiques travaillaient au bonheur de tous. Le bien est toujours l'excuse du totalitarisme. (Michel Henry, 2011, pp. 8-9)

Dans le passage précédent, le romancier cocaïne remet en cause l'interdiction et la pénalisation de l'usage des drogues. Son argumentaire pro-drogues est axé sur quatre plans : sur le plan politique, l'interdiction des drogues est synonyme de limitation de la liberté individuelle et donc de dictature de l'État ; sur le plan économique, elle est un réel manque à gagner pour l'État ${ }^{6}$; sur le plan social, elle crée de nombreuses frustrations chez les citoyens dépendants et enfin sur le plan humain, on peut noter une violation de la dignité humaine dans le traitement des personnes arrêtées et punies (détenues dans des conditions déplorables en milieu carcéral) ${ }^{7}$. Il estime en outre que c'est une

6 À ce titre il souligne en dénonçant : « [ ] cette hypocrisie [c'est-à-dire la non légalisation de la drogue] [ ] coûte des milliards d'euros à notre société, mobilise des dizaines de milliers de fonctionnaires, enferme des centaines de milliers de personnes en prison, tout en rapportant une fortune à la mafia colombienne et au terrorisme afghan. [ ] Comme dans les années 30 aux États-Unis, la prohibition des paradis artificiels n'a fait que renforcer l'économie parallèle » (Michel Henry, 2011, pp. 8-9).

7 Beigbeder en a subi un grand coup lors de sa garde à vue et sait de quoi il parle conséquemment. Aussi qualifie-t-il le Dépôt de Paris où il a douloureusement séjourné, après son transfert du Commissariat du $8^{\text {ème }}$ arrondissement de Paris (Sarij 8), de «POURRISSOIR D'HUMAINS » (Beigbeder, 2009, p. 95). 
grande perte d'énergie et de temps pour la police qui court après les consommateurs ou vendeurs de drogues. Pour lui, c'est une lâcheté de la part des policiers qui ont pourtant mieux à faire en matière des cas « sérieux » (viol, vol, meurtre, etc.). Face aux normes, Beigbeder a la posture d'une proie. Il procède à la fois à une autovictimisation et à une autojustification de son acte. C'est d'ailleurs pourquoi il est totalement d'accord avec Françoise Sagan, une pro-toxicomane, qui pense qu' « On se drogue parce que la vie est assommante, que les gens sont fatigants, qu'il n'y a plus tellement d'idées majeures à défendre, qu'on manque d'entrain » (Beigbeder, 2009, p. 43). C'est dire que la décadence qui affecte la société actuelle peut en partie se justifier par la montée de la toxicomanie. Car les individus recourent aux substances toxiques pour faire face à leur angoisse existentielle. Par contre, la société s'oppose à la toxicomanie; pourtant, elle est responsable du mal-être de l'individu. Paradoxalement, de l'avis de Beigbeder, la société détruit le citoyen mais n'arrête pas de se passer pour sa protectrice. Elle veut normaliser, formater et programmer des individus comme des automates. Or, l'individu, à l'instar de Beigbeder, un dur abusé et traqué, remet en question cette forme d'aliénation sociale. En 2011, trois ans après sa triste arrestation, le romancier rappelle: « Ce qui m’avait le plus choqué lors de ma garde à vue de 2008, c'était que des gardiens de la paix soient obligés de me séquestrer "pour mon bien"» (Michel Henry, 2011, p. 9). C'est fort à propos qu'il se considère telle une victime de l'Hexagone en affirmant : « La France avait gagné une bataille contre l'un de ses enfants » (Beigbeder, 2009, p. 113). D'après lui, il peut s'avérer parfois nécessaire de désobéir aux lois afin de préserver ses propres valeurs. La société comporte des germes de l'indignation mais elle attend plutôt de la résignation chez l'individu. Cette situation traumatisante creuse un fossé entre l'individu et la communauté. Elle tend à réduire, dégrader ou amenuiser l'homme. Le narrateur déplore ce à quoi il est réduit dans le système carcéral : « J'ai l'impression d'être redevenu le bambin que j'ai sans doute été $[. .$.$] » (Beigbeder, 2009, p. 44). Frédéric Beigbeder exprime sa révolte$ contre les règles et les normes de la société :

Pourtant la France est le pays de la liberté. Ce qui m'autorise à revendiquer le Droit de me Brûler les Ailes, le Droit de Tomber Bien Bas, le Droit de Couler à Pic. Ce sont des Droits de l'Homme qui devraient figurer dans le Préambule de la Constitution au même titre que le Droit de Tromper sa Femme sans être Photographié dans les Journaux, le Droit de Coucher avec une Prostituée, le Droit de Fumer une Cigarette en Avion ou de Boire du Whisky sur un Plateau de Télévision, le Droit de Faire l'Amour sans Préservatif avec des Personnes Acceptant de Courir ce Risque, le Droit de 
Mourir dans la Dignité Quand on est Atteint d'une Maladie Douloureusement Incurable, le Droit de Grignoter entre les Repas, le Droit de ne Pas Manger Cinq Fruits et Légumes par Jour, le Droit de Coucher avec une Personne de Seize Ans Consentante sans que Celle-ci ne Porte Plainte Cinq Ans Après pour Corruption de Mineur... Je continue ? (Beigbeder, 2009, p. 44)

Son intention est que la société, suivant la logique du libéralisme social, doit plutôt être plus tolérante, souple, voire laxiste ou anarchiste et non paternaliste ou moraliste afin de permettre beaucoup plus de liberté, voire le libertinage à l'individu. Ce n'est qu'ainsi qu'il pourra jouir de son existence libertaire, en faisant de sa vie ce qu'il veut, comme il veut et quand il le veut. C'est sans doute la raison pour laquelle il entend briser les règles et conventions en vigueur dans sa société au nom de la liberté. Il célèbre le libertinage. Force est de constater que tant sur le plan de la forme que sur le plan du fond, l'écrivain se positionne comme un rebelle, un révolutionnaire. En effet, l'emploi des majuscules dans l'énumération des droits contradictoires ou libertaires faisant l'objet de son nouveau manifeste de la L/liberté, si on peut ainsi le dire, est significatif à plus d'un titre. Autant dire qu'il s'agit du cynisme et du nihilisme visant dans certaine mesure à provoquer tout en s'indignant contre les normes trop rigides de la société, lesquelles rendent l'individu limité dans ses choix et ses actions. Selon Beigbeder, toujours dans sa préface de l'essai de Michel Henry (2011, p. 9) « [l]a prohibition crée une attirance pour l'interdit ». Alors, à son avis, l'individu doit s'affirmer, s'exprimer, jouir sans les entraves normatives de la société. C'est décidément avec une désinvolture doublée de révolte nihiliste qu'il écrit :

- Si à 42 ans je désobéis aux lois, c'est parce que je n'ai pas assez désobéi à ma mère dans ma jeunesse. J'ai 20 ans de désobéissance à rattraper. [...] Ce qui est en cause, c'est notre façon de vivre. Au lieu de frapper les victimes, demandez-vous pourquoi tant de jeunes sont désespérés, pourquoi ils crèvent d'ennui, pourquoi ils cherchent n'importe quelle sensation extrême plutôt que le sinistre destin de consommateur frustré, d'individu normalisé, de zombie formaté, de chômeur programmé. (Beigbeder, 2009, p. 44)

L'intention de la société à vouloir mouler ou standardiser l'individu et son comportement au quotidien est remise en cause totalement par le romancier libertin. II n'en revient pas ; son déphasage avec sa société est avéré. Son interrogation suivante met en lumière son profond dépit : « 24 heures de claustration pour une fiesta débile ? La société française 
devient folle! [...] Vous ne vous en rendez même pas compte mais vous cautionnez un retour à l'ordre moral complètement facho » (Beigbeder, 2009, p. 82). Il est scandalisé par la situation qui est la sienne. Sa garde à vue est pour lui une injustice notoire, scandaleuse et donc révoltante.

Force est de constater, de surcroît, que Frédéric Beigbeder fait fi du prétendu rôle que la police pense jouer pour le bien-être des citoyens français. Mieux, il faut comprendre que le cocaïnomane Beigbeder se plaît dans la cocaïnomanie. Il voudrait vivre sa vie de toxicomane en toute liberté ; sans avoir à faire face aux principes moraux et autres normes de la société française. Telle s'avère être la seule condition de son épanouissement. Dans la même perspective, son collègue et compagnon cocaïnomane, le Poète, partage la même vision que lui. Il intervient en citant un extrait révélateur d'Emmanuel Kant :

Un gouvernement fondé sur le principe de la bienveillance envers le peuple, semblable à celle d'un père envers ses enfants c'est-à-dire un gouvernement paternaliste, où donc les sujets, comme des enfants mineurs qui ne peuvent distinguer ce qui leur est véritablement utile ou nuisible, sont réduits au rôle simplement passif d'attendre du seul jugement du chef de l'État qu'il décide comment ils doivent être heureux, et de sa seule bonté qu'il veuille bien s'occuper de leur bonheur : un tel gouvernement est le plus grand despotisme qu'on puisse concevoir. (Beigbeder, 2009, p. 83)

Selon le Poète, se référant au philosophe et moraliste Kant, le gouvernement français se passe pour « un père », qui veut à tout prix inculquer «le principe de bienveillance» à « ses enfants », c'est-à-dire aux citoyens. Il s'en offusque en ce sens que le gouvernement français se veut « paternaliste », considérant les citoyens comme « des enfants mineurs » qui sont incapables de « distinguer ce qui leur est véritablement utile ou nuisible ». Le Poète et, par ricochet Beigbeder, est ainsi en désaccord avec un système politique paternaliste car trop regardant sur les actes que posent les individus. Pourtant, le bonheur recherché par les individus est relatif. Autrement dit, aucune personne physique ou morale ne saurait avoir le monopole de contribuer ou de veiller à l'épanouissement (total) de l'homme, de la société. Nous comprenons manifestement que, pour le Poète, tout comme pour Beigbeder qui partage son avis, le malheur ou le bonheur du citoyen ne devrait pas engager l'État. Car un gouvernement paternaliste est comme le souligne Kant, « le plus grand despotisme qu'on puisse concevoir ». Frédéric Beigbeder lui emboîte d'ailleurs le pas en s'inspirant d'Oscar Wilde, pour qui, « II est impossible de rendre les gens bons par décret parlementaire »(Beigbeder, 2009, p. 83). Dans la posture 
qu'adopte le Poète, il faut que l'État laisse libre cours, le "libre-arbitre », d'après l'expression cartésienne, à l'individu de vivre sa vie et de s'épanouir dans le système social sans intervention ou suivi paternaliste de la part du gouvernement.

En outre, Frédéric Beigbeder poursuit son procès social par la dénonciation des conditions drastiques de détention en France. Il entend attirer l'attention à sa son tour sur le Dépôt de Paris, après sa déplorable expérience dans cette institution pénitentiaire inhumaine et invivable :

La France a trouvé des milliards d'euros pour renflouer ses banques en 2008 mais elle tolère un POURRISSOIR D'HUMAINS au centre de Paris. Le commissaire aux droits de l'homme du Conseil de l'Europe a dénoncé cet endroit en vain. Il y a une volonté gouvernementale qui est de laisser cet endroit accablant exister au cœur de notre Cité. Quelqu'un a pris la décision rationnelle de torturer les gens en France. La France est un pays qui pratique la torture dans le ler arrondissement, juste en face de la Samaritaine. Et moi aussi je serais complice de cette calamité si je ne la décrivais pas ici. Comment ai-je pu vivre 42 années sans m'intéresser à cette atrocité qui se déroule dans ma propre ville? Comment osons-nous donner des leçons à la Chine, à I'Iran ou à la Libye alors que la France ne se respecte pas ellemême ? Nous avons élu un président de la République qui passe son temps à libérer des prisonniers à l'étranger et à jeter des gens aux oubliettes chez lui. Chers lecteurs français, des personnes présumées innocentes sont TOUS LES JOURS déférées dans ce cloaque réfrigéré et putride $A U$ PAYS DES DROITS DE L'HOMME. (Beigbeder, 2009, p. 95)

En effet, la question des Droits de l'Homme en France est loin d'être un long fleuve tranquille, selon Beigbeder. La dernière phrase de l'extrait en dit long et de manière hyperbolique et ironique; car la France n'est pas un paradis à ses yeux. Son passage au Dépôt lui a permis de se rendre compte des réalités physiques et psychologiques de ce pénitencier. L'enjeu de son vitriol est de faire connaître au grand jour les atrocités subies par la population carcérale dans un pays de droit comme la France. D'après l'écrivain, sa patrie est, en réalité, un pseudo " pays des Droits de l'Homme », vu la réalité carcérale par lui dénoncée avec véhémence. Beigbeder s'inscrit en vrai avec Michel Foucault qui, dans Surveiller et punir : naissance de la prison, remet en cause la logique de surveillance organisée à visée « normalisatrice » qui semble prévaloir dans les sociétés (post)modernes. Selon ce philosophe, «Le châtiment est passé d'un art des sensations 
insupportables à une économie des droits suspendus » (Foucault, 1975, p. 18). Sur le plan individuel, Beigbeder s'offusque à cause de préjudice ou de la torture subi(e) pour avoir consommé de la cocaïne. II rejette en brèche la moralité qui fonde ou justifie l'épreuve de sa punition si dégradante. Selon lui, sa dignité et sa liberté ont été violées, sous prétexte que l'État à travers la police l'empêche de se livrer à une pratique d'autodestruction qu'est la consommation de drogue. C'est fort de ce constat qu'il affirme avec un dépit profond : «Les principes moraux qui vous ont conduit à m'infliger cette violence [physique et psychologique] sont mille fois moins importants que ceux que vous venez de bafouer [...]» (Beigbeder, 2009, p. 112).

Dans une interview accordée à Jérôme Colin à Bruxelles, un an après sa garde à vue dans une «cellule crasseuse », le romancier défend la liberté de l'autodestruction :

Jérôme : Vous êtes pour la liberté de se détruire si on en a envie? Frédéric Beigbeder : Exactement.

Jérôme : La liberté de chacun de décider.

Frédéric Beigbeder : Oui, et je crois qu'il y a une dérive un peu bizarre, notamment en France, sur le plan des libertés, parce que maintenant tout est interdit, quasiment tout est interdit. C'est pour ça que j'adore regarder une série américaine qui s'appelle « Mad Men » où on voit des gens dans les années 60 , l'époque où tout était permis. Je fantasme beaucoup sur les années 60 . Je crois qu'on n'a pas de chance d'être né dans les années où les libertés rétrécissent. Enfin, c'est cyclique, ça reviendra. (Jérôme Colin, 2010, p. 9)

Les années 60 sont évoquées par le romancier car le leitmotiv du Mouvement de Mai 68 en France était bien « Jouir sans entraves ». Telle est la philosophie de la vie qui semble sied le mieux à Beigbeder, né curieusement dans les années 70 marquées, en France, par la libération sociale et sexuelle. D'ailleurs, il exprime son regret d'avoir pas eu la possibilité de vivre à cette époque plus libérale que celle actuelle, la sienne. Mais, il est loin d'être pessimiste : il garde espoir que la belle époque, d'après lui, « reviendra ». Cette projection dans un futur euphorique est un refuge, une source de consolation qui redonne le goût de vivre, voire de survivre chez Beigbeder.

Somme toute, Frédéric Beigbeder est en désaccord complet avec la logique de sa société, notamment en ce qui concerne l'interdiction des drogues. Il est contre le paternalisme et pour le libéralisme social ; car il est individualiste et hédoniste. Dans 
la conclusion de sa préface pro-légalisation/libéralisation des drogues, il souligne la « fragilité de l'humanité face aux addictions » et l'« inefficacité des atteintes à sa liberté » (Michel Henry, 2011, p. 10). C'est dire que les humains sont des potentiels sujets aux comportements addictifs; et, en même temps, on ne saurait tolérer les atteintes à la liberté des individus de disposer de leur vie. Beigbeder s'explique à ce propos, en faisant le constat suivant sur la question des drogues:

Plusieurs degrés existent dans la légalisation : certains pays ont dépénalisé la drogue douce, d'autres ont cessé de poursuivre les simples usagers, d'autres encore tentent de mieux contrôler le commerce (coffee-shops) ou d'améliorer la qualité de la consommation (salles d'injection, tests médicaux en " raves », etc.). II faut faire preuve d'imagination dans ce domaine où le puritanisme empêche toute décision rationnelle : le législateur est souvent dopé à la moraline ${ }^{8}$, cette drogue l'empêchant de travailler en toute objectivité. (Michel Henry, 2011, p. 10)

Autant dire que Frédéric Beigbeder invite tacitement les législateurs à revoir « rationnellement » et « objectivement » le cadre réglementaire en matière de drogues dans sa société. Peut-être, faut-il espérer, comprendront-ils enfin « pourquoi la légalisation est inévitable », pour reprendre le sous-titre révélateur de la thèse de Michel Henry (2011) dans son essai Drogues ${ }^{9}$.

\section{Conclusion}

Le cadre social demeure un théâtre de tensions et d'aspirations à la fois pour des intérêts individualistes et pour ceux collectifs. Cette relation dialectique, sous-tendue par plusieurs mobiles, se déploie suivant plusieurs modalités. Le cas de Frédéric Beigbeder en divorce avec sa société, notamment suite à son arrestation et son incarcération pour consommation de drogue, illustre le fait que l'individu, par pur égoïsme et orgueil inhérents à la nature humaine, a tendance à opérer des choix privés et à se comporter

8 Ce mot a pour substantif « moral- ». L'auteur souligne(rait) et dénonce(rait) ainsi l'approche quelque peu moralisante du dispositif normatif qui semble s'inspirer du principe de "la bonne moralité » d'origine chrétienne, voire religieuse. Beigbeder, ironiquement, s'inscrit sans doute en faux par rapport à cette philosophie du droit qui tend à ériger les législateurs en des prescripteurs de « la bonne moralité » à suivre par les citoyens.

9 "Comme les jeux d'argent ou la prostitution, la consommation de drogues est une activité humaine que rien n'arrêtera, et surtout pas les leçons de morale. Face à la banalisation de l'usage, l'interdit n'est plus applicable, et cela discrédite la loi qui l'édicte », soutient Michel Henry (2011, p. 15). Lire aussi à ce sujet Nicole Maestracci, Les Drogues, PUF, 2005. 
de manière transgressive, au détriment de la société. Dès lors, on note une rupture, un divorce net entre le microcosme qu'est le sujet individuel et le macrocosme qu'est le sujet social. Ainsi, le narrateur-héros-auteur Frédéric Beigbeder procède, dans son autobiographie, à une mise en scène de soi sous le prisme des réalités sociales auxquelles il est confronté. Il s'avère que Beigbeder est un individu libertaire, individualiste et hédoniste. Il part de son expérience carcérale, douloureusement vécue, pour dénoncer les conditions de détention en France, tout en exprimant son dédain à l'égard de son pays natal, sous un ton résolument pamphlétaire. Alors, il va sans dire que le romancier est en déphasage complet avec sa France ultra-capitaliste, paternaliste et moraliste, qui est proprement pathologique et donc angoissante, voire aliénante. Aussi faut-il rappeler que la France est ciblée par l'auteur à partir de son titre thématicométhaphorique : Un roman français. Beigbeder, parlant de son soi individuel en rapport étroit avec le soi social, parvient à faire un procès de sa France natale qui est, selon lui, une société liberticide. Car elle étouffe ou limite l'épanouissement de l'individu pris au piège d'une prétendue « liberté ». De l'avis de l'écrivain, son pays n'est pas une terre de L/liberté. À la lecture de ce titre, la vie de Frédéric Beigbeder serait le miroir (plus ou moins brisé) de la société française. L'écrivain se perçoit sans doute comme un produit pur et dur de sa société : il fait une corrélation entre sa vie, son histoire personnelle et l'expérience collective. Alors, il serait donc un parangon du «sujet culturel» français (Edmond Cros, 2005). Autrement dit, le projet autobiographique de Beigbeder problématise le social à partir de l'individuel. C'est ce que Annie Ernaux $(1994 ; 2011)$ nomme « l'autosociobiographie », qui s'opère à travers un « je[u] transpersonnel », que nous nommons à sa suite de l'autosociobiographisme.

\footnotetext{
Évaluation : Évaluation anonyme par des pairs extérieurs.

Conflit d'intérêts : L'auteur n'a aucun conflit d'intérêts à déclarer.

Subvention : L'auteur n'a reçu aucun soutien financier pour ce travail.
}

Peer-review: Externally peer-reviewed.

Conflict of Interest: The author has no conflict of interest to declare.

Grant Support: The author declared that this study has received no financial support.

\section{Bibliographie}

Aristote, Barthelemy-Saint-Hilaire J. (Trad.) (1874). La Politique. Wikisource.

Baudelaire C. (2001). Les Fleurs du mal. Mozambook [édition numérique].

Beigbeder F. (2009). Un roman français. Paris, FR : Grasset \& Fasquelle. 
Colin J. (2010). Frédéric Beigbeder dans le Taxi de Jérôme Colin [interview]. en ligne : https://ds1.static.rtbf.be/ article/pdf/beigbeder-1378374822.pdf.

Cros E. (2005). Le Sujet culturel. Sociocritique et psychanalyse. Paris, FR : L'Harmattan.

Ernaux A. (1994). Vers un je transpersonnel. RITM (6), 219-222.

Ernaux A. (2011 [2003]). L'Écriture comme un couteau. Entretien avec Frédéric-Yves Jeannet. Paris, FR : Gallimard.

Foucault M. (1975). Surveiller et punir. Naissance de la prison. Paris, FR : Gallimard.

Genette G. (1972). Figure III. Paris, FR: Seuil.

Henry M. (2011). Drogues. Pourquoi la légalisation est inévitable. Paris, FR : Éditions Denoël.

Hobbes T., Folliot P. (trad.) (2002 [1651]). Léviathan. Les classiques des sciences sociales,http://www.uqac. uquebec.ca/zone30/Classiques_des_sciences_sociales/index.htmlLipovetsky G. (1983). L'Ère du vide. Essais sur l'individualisme contemporain. Paris, FR : Folio.

Maestracci N. (2005). Les Drogues, Paris, FR: PUF.

Meizoz J. (2011). La Fabrique des singularités. Postures littéraires II. Genève, SU : Slatkine Érudition.

Proguidis L. (2001). De l'autre côté du brouillard. Essai sur le roman français contemporain, Québec, CA : Nota bene. Rousseau J-J. (2012 [1762]). Du contrat social, ou principes du droit politique. in Collection complète des œuvres,

Genève, 1780-1789, vol. 1, in-4, édition en ligne www.rousseauonline.ch, version du 7 octobre 2012. Consulté le 15 juillet 2019.

Stuart Mill J. (1990 [1859]). De la liberté. Paris, FR : Gallimard. 
